The first chapter, which is devoted to 'Hepatitis', contains some very low key statements such as '... the surgeon plays a critical role when medical therapy is no longer possible ...' and 'The clinical immunologist is responsible for performing the many immunologic assays required to identify and characterise the different forms of chronic hepatitis.' This chapter might be satisfactory for undergraduate teaching but is not detailed enough for trainee specialists. In this and in some of the subsequent chapters it was not always clear which readership the editors had in mind when they started the project.

Chapter two, on hepatic failure, is also rather disappointing. A discussion of pathogenesis is confined to just one column and the text includes unhelpful phrases such as 'Transaminases are usually elevated, often dramatically', and 'Thus the transplant team needs to be involved in a timely way for those patients deemed to be candidates'. These comments again suggested that the editors had not defined the level of expertise they expected from their readership and this was again evident in chapter 4 on portal hypertension. In this chapter there is considerable emphasis on the technique and indications for transjugular intrahepatic portosystemic shunt (TIPS) procedures but little on the details of more conventional surgical procedures. There is no analysis of the problems of extrahepatic portal hypertension in which neither TIPS or transplantation are options, and no discussion on the value of devascularisation procedures in the bleeding patient.

The concept of inviting such a large number of contributors was very ambitious but this may have been the cause of some of the inconsistencies. Drug dosages, for example, are detailed in some chapters (hepatic failure), but are completely absent in others (portal hypertension). Surgical techniques are described extensively in some sections (hydatid disease) but are absent in others. Most of the important inconsistencies are found in the first few chapters and if the book was intended for fellow hepatologists then in my opinion the data (and the references) should have been much more complete.

However, the later chapters, especially those concerning pancreatic disease, are very good overviews on management and I particularly liked those on pancreatitis. Therefore despite my reservations I think that the book can be recommended as a useful addition to the library of the trainee gastroenterologist or surgeon.

E R HOWARD

Longitudinal Muscle in Esophageal Disease. By O Arthur Stiennon. (Pp 300; illustrated; \$150.) Maddison, Wisconsin: WRS Press, 1995. ISBN 0-9644 594-0-X.

This is an interesting book that I enjoyed reading, because unlike $99 \%$ of medical textbooks, the author challenges conventional views and makes the reader think. I am certainly persuaded that the function of the oesophageal muscle has been largely ignored, compared with other components thought to play a part in oesophageal disease and the radiological evidence of longitudinal muscle function, seems quite compelling.

The book is written in dogmatic style, rather like a lawyer making a case for the prosecution. $\mathrm{He}$ chooses to ignore the 'defence' evidence and this is both the strength and weakness of the text. The basis of Dr Stiennon's hypothesis is that the longitudinal muscle of the oesophagus is somehow responsible for just about every benign oesophageal disorder. I suspect that he is right about longitudinal muscle function and the subsequent effects of oesophageal shortening. Everything seems fine until the 'lawyer' has to sum up for the jury at the end of each chapter. It is here that the author strays from the evidence and in particular that which does not fit with his theory. The chapter on Barrett's oesophagus is particularly short sighted and uses the most tenuous literature review to support the author's notion that this is yet another condition explained entirely by longitudinal muscle action and that the Barrett's segment is no more than a cylinder of stomach, pulled up by the longitudinal muscle of the oesophagus.

While I agree with the author's scepticism regarding current theories of the pathogenesis of achalasia, I find them no more bizarre than his interpretation that diffuse oesophageal spasm and achalasia are manifestations of undiagnosed obstruction due to incarceration of the gastric fundus. I would be interested to know how patients with diffuse oesophageal spasm get their symptoms at night, when motor function is largely quiescent. It is when the author strays into the field of surgery, that he really gets my back up. Most of his comments about anti-reflux surgery and surgery for achalasia, are so misplaced that one wonders if he has ever read any of the surgical literature on the oesophagus in the past 40 years! There is no shortage of literature for instance, testifying to the poor performance of the Allison repair in the long term and by $\mathrm{Dr}$ Stiennon's theories, all Nissen fundoplications should result in gasbloat syndrome. No explanation is offered why over $95 \%$ of these operations do not produce the syndrome.

This book should be read by 'oesophagologists', if only to challenge their existing views. I cannot recommend it, however, to a wider audience as a balanced account of the role of the longitudinal muscle in the pathogenesis of oesophageal disease. The author claims that many of our beliefs about oesophageal function and disease, stem from preconceived notions. He uses the analogy of 'The Flat Earth Society' to condemn these views as two dimensional in a three dimensional organ. I would remind readers, however, of $\mathrm{T} \mathrm{H}$ Huxley's aphorism, that 'The great tragedy of science is the slaying of a beautiful hypothesis by an ugly fact'. At the moment, there are too many ugly facts to support Dr Stiennon's hypothesis.

D A ALDERSON

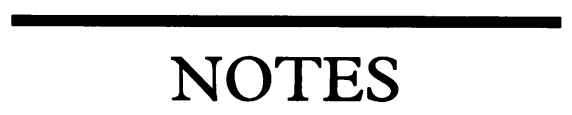

Digestive endotherapy

The XIVth European Workshop on Digestive Endotherapy will be held on 18-20 June, 1996 in Brussels. Further information from: Administrative Secretariat, Conference Services sa, Avenue de l'Observatoire, 3, Box
17, B-1180 Brussels, Belgium. Tel: (32) 2 37516 48; fax: (32) 23753299 .

\section{Abdominal imaging}

A course on Abdominal Imaging will be held on 1-5 July 1996 in Wyoming, USA. Further information from: Janice Ford Benner, University of Pennsylvania Medical Center, 3400 Spruce Street, 1 Silverstein Building, Philadelphia, PA 19104, USA. Tel: (215) 662-6904; fax: (215) 349-5925.

\section{Gastrointestinal pharmacology}

A meeting sponsored by the Gastrointestinal (GI) Pharmacology Section of IUPHAR (International Union of Pharmacology) will be held 3-7 September 1996 in Sperlonga, Italy. The meeting is entitled New Approaches to Pharmacotherapy for Gastrointestinal Ulcerative and Inflammatory Disorders. Further information from: Dr Antonio Guglietta, Parke-Davis Research, 2800 Plymouth Road, Ann Arbor, MI, 48105, USA. Tel: (313)-996-2911; fax: (313)-996-4333; e-mail: gugliea@aa.wl.com.

\section{Clinical nutrition}

The Leeds Course in Clinical Nutrition will be held on 3-6 September 1996. Further details from: Mrs Hilary L Thackray, Department of Continuing Professional Education, Continuing Education Building, Springfield Mount, Leeds LS2 9NG. Tel: 0113233 3233; fax: 01132333240 .

\section{Inflammatory bowel disease}

An International Meeting on Inflammatory Bowel Disease will be held in Capri, Italy, on 19-21 September 1996. Further information from: Professor Renzo Caprilli, Cattedra di Gastroenerologia, Università de L'Aquila, Via S Sisto 22E 67100 L'Aquila, Italy. Tel: 39 862 433407; fax: 39862433383.

\section{CORRECTION}

\section{Correction}

An authors' error occurred in the paper by $\mathrm{Dr}$ R J Scott and others (Gut 1995; 36: 731-6). The mutation described at nucleotide 5934 codon 1978 should be nucleotide 5960, codon 1987 causing a stop codon 61 codons later as shown in the accompanying Figure.

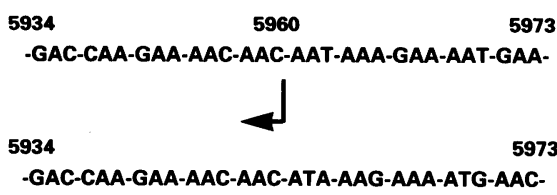

Figure 3: Sequence analysis of exon 15n showed a base pair deletion at position 5960, which was identified in patients who presented with colonic disease alone or extracolonic disease with or without colonic disease. 